\title{
Optimized T1- and T2-weighted volumetric brain imaging as a diagnostic tool in very preterm neonates
}

\author{
Revital Nossin-Manor • Andrew D. Chung • Drew Morris • João P. Soares-Fernandes • \\ Bejoy Thomas • Hai-Ling M. Cheng • Hilary E. A. Whyte • Margot J. Taylor • \\ John G. Sled • Manohar M. Shroff
}

Received: 30 July 2010 /Revised: 1 November 2010 /Accepted: 12 November 2010 /Published online: 16 December 2010

(C) Springer-Verlag 2010

\begin{abstract}
Background T1- and $\mathrm{T}_{2}-\mathrm{W}$ MR sequences used for obtaining diagnostic information and morphometric measurements in the neonatal brain are frequently acquired using different imaging protocols. Optimizing one protocol for obtaining both kinds of information is valuable.

Objective To determine whether high-resolution T1- and $\mathrm{T}_{2}-\mathrm{W}$ volumetric sequences optimized for preterm brain imaging could provide both diagnostic and morphometric value.

Materials and methods Thirty preterm neonates born between 24 and 32 weeks' gestational age were scanned during the first 2 weeks after birth. T1- and $\mathrm{T}_{2}-\mathrm{W}$ highresolution sequences were optimized in terms of signal-to-
\end{abstract}

R. Nossin-Manor $(\bowtie)$

Department of Diagnostic Imaging,

The Hospital for Sick Children,

Neurosciences \& Mental Health, Research Institute,

555 University Ave.,

Toronto M5G 1X8, Canada

e-mail: rmanor@phenogenomics.ca

A. D. Chung $\cdot$ D. Morris $\cdot$ J. P. Soares-Fernandes $\cdot$ B. Thomas $\cdot$

M. M. Shroff

Department of Diagnostic Imaging,

The Hospital for Sick Children,

Toronto, Canada

J. P. Soares-Fernandes

Neuroradiology Department,

Hospital de S. Marcos,

Braga, Portugal

\section{H.-L. M. Cheng}

Department of Diagnostic Imaging,

Physiology Experimental Medicine, Research Institute,

The Hospital for Sick Children,

Toronto, Canada noise ratio, contrast-to-noise ratio and scan time and compared to conventional spin-echo-based sequences.

Results No differences were found between conventional and high-resolution $\mathrm{T}_{1}-\mathrm{W}$ sequences for diagnostic confidence, image quality and motion artifacts. A preference for conventional over high-resolution $\mathrm{T}_{2}-\mathrm{W}$ sequences for image quality was observed. High-resolution T1 images provided better delineation of thalamic myelination and the superior temporal sulcus. No differences were found for detection of myelination and sulcation using conventional and high-resolution $\mathrm{T}_{2}-\mathrm{W}$ images.

Conclusion High-resolution T1- and $\mathrm{T}_{2}$-W volumetric sequences can be used in clinical MRI in the very preterm brain to provide both diagnostic and morphometric information.

\section{H.-L. M. Cheng}

Medical Biophysics Department, University of Toronto,

Toronto, Canada

H. E. A. Whyte

Neonatology Department, The Hospital for Sick Children,

Neurosciences \& Mental Health, Research Institute,

Toronto, Canada

\section{J. Taylor}

Neurosciences \& Mental Health, Research Institute,

Department of Diagnostic Imaging,

The Hospital for Sick Children,

Toronto, Canada

M. J. Taylor

Medical Imaging, University of Toronto,

Toronto, Canada

\section{J. G. Sled}

Physiology Experimental Medicine, Research Institute,

The Hospital for Sick Children, Medical Biophysics,

University of Toronto,

Toronto, Canada 
Keywords MRI $\cdot$ Preterm brain $\cdot$ Neonates .

T1- and T2-weighted imaging - Relaxation times .

Myelination $\cdot$ Sulcation

\section{Introduction}

MRI has become the modality of choice for investigating normal brain maturation as well as neonatal brain injury and developmental neurological disorders because of the modality's superior soft-tissue contrast, good spatial resolution and various physical parameters that can be used as contrast mechanisms [1-12]. The development of MR-compatible incubators and dedicated neonatal imaging coils has further enabled safe and fast acquisition of high-resolution anatomical and functional imaging of the neonatal brain with greater signal-to-noise ratio (SNR) [12]. However, image contrast and signal intensity changes occurring during the first year of life necessitate careful optimization of the experimental parameters used in neonatal MRI [13-18]. In neonates, image contrast is inverted relative to adults [19-22], as relaxation times of white matter (WM) are longer than those of gray matter (GM) $[15,23]$. Decreases in brain water content, neuronal and synaptic development and increases in the concentration of macromolecules associated with myelin cause $\mathrm{T} 1$ and $\mathrm{T} 2$ relaxation times to decrease significantly during the first 2 years $[1,2,5,8,18]$. As optimal TRs and TEs used for $\mathrm{T} 1-$ and $\mathrm{T}_{2}-\mathrm{W}$ sequences are based on the values of $\mathrm{T} 1$ and $\mathrm{T} 2$, appropriate values for adults and even 2-year-olds might not be suitable for neonates [13, 15, 18]. Therefore, both SNR and GM-WM contrast in neonates should be optimized according to age-appropriate relaxation times. Although cerebral T1 and T2 relaxation measurements have been reported at various field strengths $(1.0-3.0 \mathrm{~T})$ for neonates born at term and for preterm infants at term [15, 23-28], little has been reported on these values in the preterm brain at birth [24, 28, 29], with no reports at $1.5 \mathrm{~T}$.

$\mathrm{T}_{1}-\mathrm{W}$ and $\mathrm{T}_{2}-\mathrm{W}$ images are used routinely for monitoring development and identifying pathologies in the neonatal brain $[2,4-14,18]$. These images are also used for segmentation and quantitative morphometric measurements in the developing brain [14, 17, 30-37]. Frequently, however, sequences used for clinical diagnosis and morphometric measurements are acquired using different imaging protocols and experimental settings. While clinical scanning often involves spin-echo (SE) $\mathrm{T}_{1}-\mathrm{W}$ and $\mathrm{T}_{2}-\mathrm{W}$ sequences, having limited spatial resolution in one of the imaging directions $[6,9-11,18]$, isotropic high-resolution imaging is increasingly used for morphometric studies [7, 11, 31, 34]. Developing appropriate sequences is almost always a trade-off between increas- ing scan time to improve scan quality and risking image degradation caused by patient movement. Limited scan time and the potential for motion artifacts are major issues in neonatal imaging, especially when the patients are not sedated. In today's clinical research there is a growing need to obtain diagnostic information as well as morphometric data. The purpose of this study was to optimize the experimental parameters of $\mathrm{T}_{1}-\mathrm{W}$ and $\mathrm{T}_{2}-\mathrm{W}$ high-resolution (isotropic) volumetric sequences used in very preterm neonates (younger than 32 weeks' gestation), and compare these to conventionally used SE sequences in terms of SNR, contrast-to-noise ratio (CNR), diagnostic confidence, image quality and motion artifacts to determine whether high-resolution $\mathrm{T}_{1}-\mathrm{W}$ and $\mathrm{T}_{2}-\mathrm{W}$ volumetric sequences can provide both diagnostic and morphometric value. $\mathrm{T} 1$ and $\mathrm{T} 2$ relaxation times of the very preterm brain are provided.

\section{Materials and methods}

\section{Patients}

The study included 30 preterm neonates showing a range of pathologies born between 24 to 32 weeks' gestational age (median 29.00 weeks) and scanned between 26 to 34 weeks' postmenstrual age (median 30.57 weeks). The median birth weight of the neonates was $1,246 \mathrm{~g}$ (range 728-2,070 g). All were scanned within the first 2 weeks after birth. None had evidence of a genetic, metabolic or viral infection disorder. All neonates but two were scanned without sedation. These children are part of a broader cohort of a prospective 4-year neuroimaging study approved by the hospital's research ethics board. Informed, written consent was given by the neonates' parents.

\section{MR acquisition}

MR scans were performed on a 1.5-T Signa Excite HD scanner (GE Medical Systems, Milwaukee, WI, USA) using an MR-compatible incubator and neonatal head coil (AIR Inc., Cleveland, OH, USA). MRI protocol and the associated MR parameters, scan times, SNR and CNR are summarized in Table 1. Conventional clinical sequences will be referred to throughout the paper as clinical sequences whereas high-resolution sequences will be referred to as research sequences. For the research $\mathrm{T}_{1}-\mathrm{W}$ sequence, $\mathrm{T}_{1}$ values of $1,700 \mathrm{~ms}$ for $\mathrm{WM}$ and $1,200 \mathrm{~ms}$ for GM were used based on data from previous term literature [15] in order to estimate the appropriate TR/flip angle (FA) combination. A TR/FA combination of $23 \mathrm{~ms} / 19^{\circ}$ yielded the shortest scan time for $1-\mathrm{mm}^{3}$ resolution $\mathrm{T}_{1}-\mathrm{W}$ volume with an SNR of 22.1 and $\mathrm{CNR}$ of -6.4. A $\mathrm{T}_{2}$ value of 


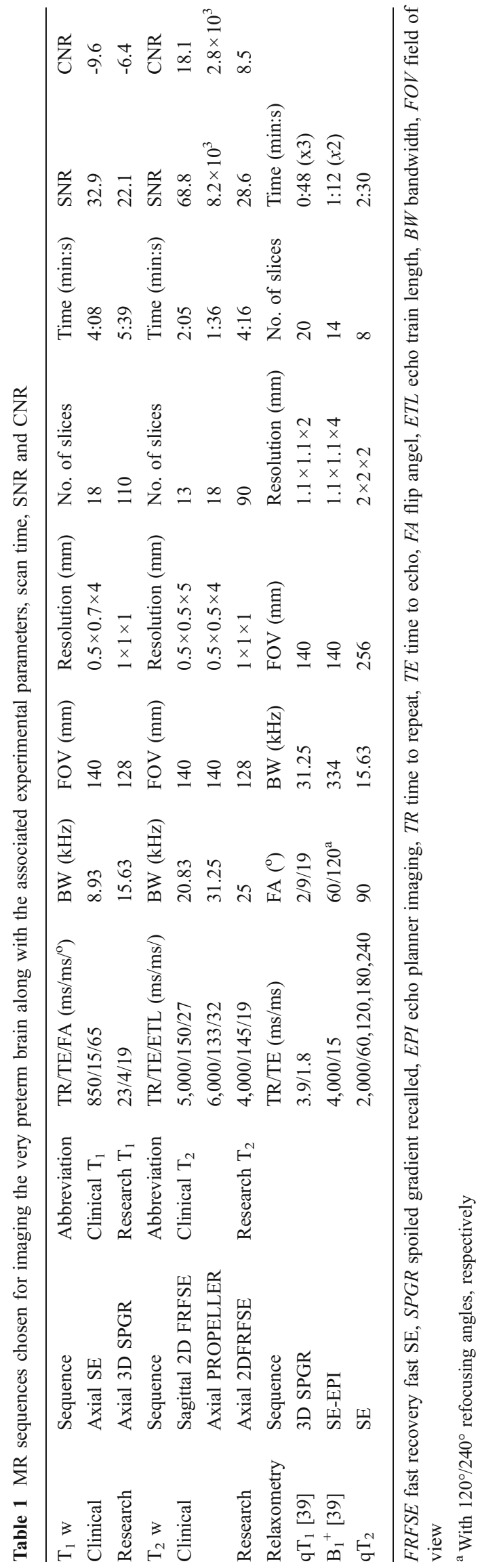

$200 \mathrm{~ms}$ was estimated for determining the TE for the research $\mathrm{T}_{2}$ sequence [15]. Therefore, using TR of 4,000 ms, TEs of 145, 158 and $200 \mathrm{~ms}$ were tested. All TEs produced images having a comparable SNR of about 28 and CNR of about 13; thus to achieve the shortest scan time possible for $1 \mathrm{~mm}^{3}$ resolution a TE of $145 \mathrm{~ms}$ was chosen. Both SNR and CNR were calculated considering the Rayleigh distribution for noise in image regions with no NMR signal [38]:

$S N R=\frac{S_{W M}}{\sigma_{M} / 0.655}$

$C N R=\frac{S_{W M}-S_{G M}}{\sigma_{M} / 0.655}$

where $\mathrm{S}_{\mathrm{WM}}$ represents the mean signal intensity in regions of interest (ROIs) drawn in the frontal, periventricular and posterior WM (SNR and CNR represent an average of three separate measurements in three patients), $\mathrm{S}_{\mathrm{GM}}$ represents the mean signal intensity in the frontal cortical GM and $\sigma_{\mathrm{M}}$ is the standard deviation of the background signal of the magnitude image.

Quantitative T1 (qT1) and T2 (qT2) maps were obtained for six and four neonates, respectively, using the relaxometry protocol provided in Table 1 . The inclusion criteria for these cases included germinal matrix hemorrhage (GMH) grade I, small ischemic focus and no abnormalities at all. T1 parametric maps were produced using a linear least squares solution according to the methodology presented by Cheng and Wright [39]. For accurate flip angle calculation, a rapid $\mathrm{B}_{1}{ }^{+}$mapping sequence was employed. T2 maps were obtained by fitting the four data points into a monoexponential function at each voxel using a non-linear least squares fit. Mean T1 and T2 values were calculated from manually bilaterally drawn ROIs in the frontal WM, periventricular WM, posterior WM, frontal cortical GM and posterior cortical GM (selected at the level of the lateral ventricles), and manually segmented structures of the basal ganglia and thalami.

\section{Radiological assessment}

Four neuroradiologists experienced in neonatal imaging independently evaluated each of the clinical and research sequences separately. Radiologists evaluated the clinical images for all neonates and then rated the research images. Raters completed a questionnaire noting detected lesions by type and rating the sequences using a five-level Likert scale for diagnostic confidence, image quality and presence of motion artifacts. In addition, the presence of selected myelination (dorsal brainstem, thalami and cerebellar peduncles) and sulcation (insular, central, parieto-occipital, 

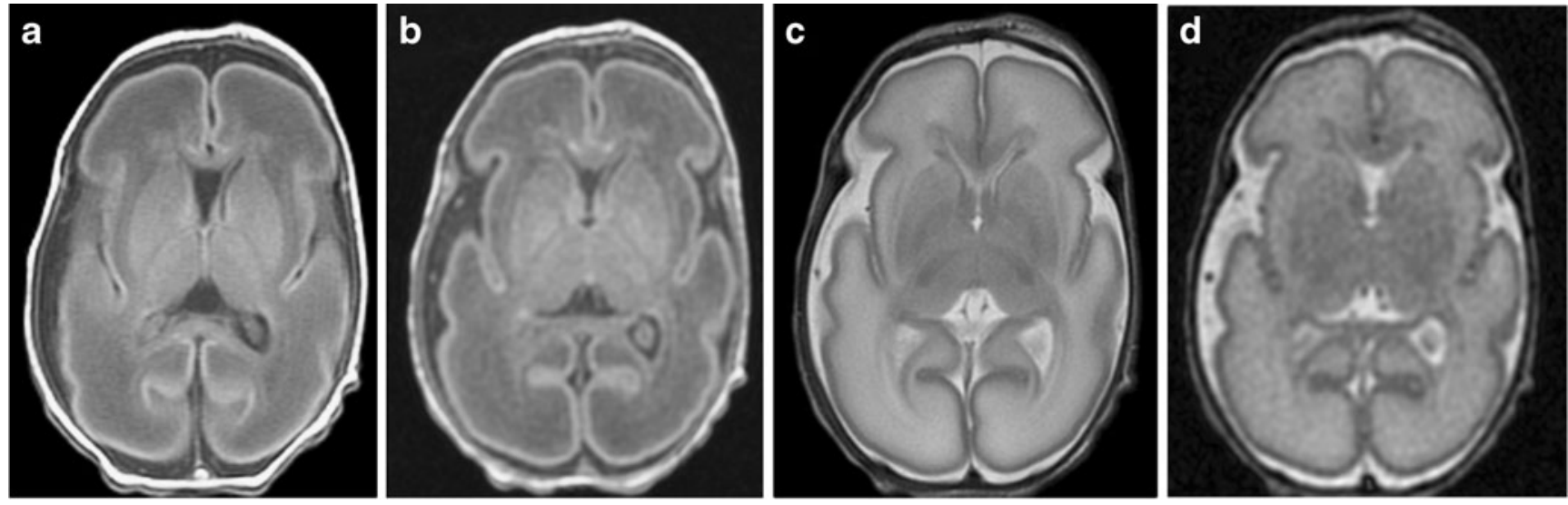

Fig. 1 Clinical and research $T_{1}-W$ and $T_{2}-W$ representative images in the axial plane of a neonate born at 29 weeks and scanned at 30.57 weeks: (a) clinical T1, (b) research T1, (c) clinical T2 (PROPELLER), (d) research T2

superior temporal and calcarine sulcus) milestones on both clinical and research images were evaluated using a binary scale where 0 meant not present and 1 meant present.

\section{Statistical analysis}

Paired $t$-tests were conducted to determine whether there were significant differences between relaxation times measured in the right and left hemispheres for each anatomical region. The Kolmogorov-Smirnov (K-S) test was used to determine whether the distributions of the combined rating results for the clinical and research sequences were significantly different. Combined ratings were calculated by summing individual rater scores on a case-by-case basis for each metric and counting the number of cases for each result. K-S tests were performed for rating diagnostic confidence, image quality and motion artifacts as well as for the results of myelination and sulcation. Interrater agreement for diagnostic confidence, image quality and motion artifacts was evaluated using the Cronbach alpha, where alpha $>0.7$ was considered to indicate high agreement among the four raters. Inter-rater agreement for myelination and sulcation was evaluated by computing a free-marginal kappa, where $\kappa>0.7$ was considered highly reliable. Statistical analyses were performed using R (www. r-project.org) and SPSS (SPSS Inc., Chicago, IL, USA).

\section{Results}

Figure 1 shows clinical and research $T_{1}-W$ and $T_{2}-W$ representative axial images, corresponding to the chosen sequences presented in Table 1, of a preterm neonate born at 29 weeks and scanned at 30.57 weeks. Figure 2 displays qT1 and qT2 maps at the level of the basal ganglia of a preterm neonate born at 28 weeks, scanned at 29 weeks. Table 2 presents the mean relaxation times measured in seven anatomical regions. Statistical analysis showed no significant differences between measurements taken at the left and right hemispheres for both $\mathrm{T} 1$ and $\mathrm{T} 2$ values. Therefore, left and right values were averaged. Also, as averaged rather than regional relaxation times are used for sequence optimization, averaged relaxation times were calculated for the WM and GM.

Clinical and research $\mathrm{T}_{1}$ sequences demonstrated similar combined rating distributions $(P>0.05)$ for diagnostic confidence, image quality and motion artifacts (Fig. 3, Table 3). A trend preferring research $T_{1}$ for diagnostic confidence (Fig. 3, $P=0.11$ ) and a slight trend favoring clinical T1 for image quality and motion artifacts were observed. All three measures demonstrated high inter-rater agreement (Cronbach alpha $=0.71-0.87$ ) for both clinical and research sequences, with the exception of diagnostic confidence for research $\mathrm{T} 1$ (Cronbach alpha=0.58). Re-
Fig. 2 T1 (a) and T2 (b) maps at the level of the basal ganglia of an infant born at 28 weeks, scanned at 29 weeks, shows no abnormalities on conventional MRI
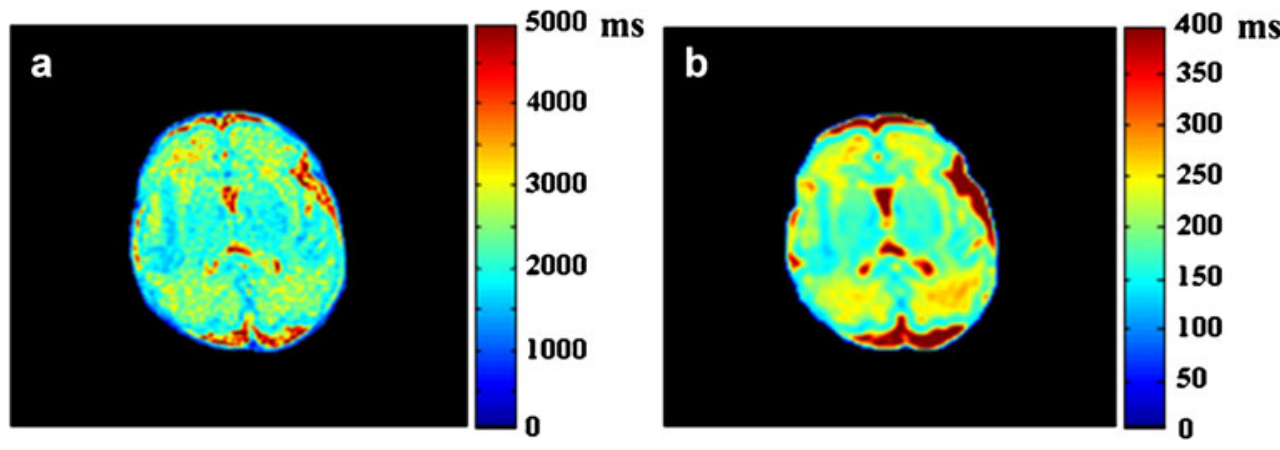
Table $2 \mathrm{~T} 1$ and T2 relaxation times at various anatomical regions at WM and GM areas in the very preterm brain

\begin{tabular}{lcc}
\hline Anatomical region & $\mathrm{T} 1(\mathrm{~ms})^{\mathrm{a}}(n=6)$ & $\mathrm{T} 2(\mathrm{~ms})^{\mathrm{a}}(n=4)$ \\
\hline Frontal WM & $2,863 \pm 99$ & $216 \pm 7$ \\
Periventricular WM & $2,839 \pm 55$ & $230 \pm 4$ \\
Posterior WM & $3,045 \pm 139$ & $255 \pm 4$ \\
WM-average & $2,916 \pm 65$ & $234 \pm 7$ \\
Frontal cortical GM & $2,064 \pm 52$ & $153 \pm 4$ \\
Posterior cortical GM & $1,975 \pm 37$ & $157 \pm 4$ \\
Basal ganglia & $2,196 \pm 41$ & $167 \pm 5$ \\
Thalami & $2,162 \pm 43$ & $158 \pm 2$ \\
GM-average & $2,100 \pm 50$ & $159 \pm 3$ \\
\hline
\end{tabular}

${ }^{\mathrm{a}}$ Values are given as mean \pm standard error

search $\mathrm{T}_{1}$ provided significantly better detection for thalamic myelination (Fig. 4) while similar rating distributions were found for the dorsal brainstem and cerebellar peduncles. Nevertheless, a trend toward better detection of myelination in the latter on research $\mathrm{T} 1$ is seen in Fig. 4. High inter-rater agreement was established for dorsal brainstem in both clinical and research $\mathrm{T} 1$ sequences $(\kappa=$ 0.89 and 0.93 , respectively). However, only fair $(\kappa \leq 0.40)$ and moderate $(0.40<\kappa \leq 0.60)$ agreement between raters was found for clinical and research T1 sequences, respectively, for the thalami and cerebellar peduncles. For sulcation, research $\mathrm{T} 1$ provided significantly better detection of the superior temporal sulcus (Fig. 5); a trend toward better detection of the calcarine sulcus was observed (Fig. 5). Otherwise, no significant differences were found between clinical and research $T_{1}$ for sulcus detection in the preterm brain. Inter-rater agreement was high $(\kappa>0.7)$ for all five sulci identified on research $\mathrm{T} 1$ and the insular, central and parieto-occipital sulci on clinical T1. Moderate agreement was found for the superior temporal and calcarine sulci on clinical $\mathrm{T} 1 \quad(\kappa=0.49$ and 0.61 , respectively), indicating better detection and improved consistency between raters when using research $\mathrm{T} 1$.

For $\mathrm{T}_{2}-\mathrm{W}$ images, combined rating results (Fig. 3) and $\mathrm{K}-\mathrm{S}$ test (Table 3) demonstrated a clear preference for clinical over research sequences for image quality $(P=$ 0.006), while similar combined rating distributions were found for diagnostic confidence and motion artifacts. Ratings for image quality and motion artifacts showed high inter-rater agreement (Cronbach alpha $=0.76-0.86$ ) for both sequences, while fair agreement between raters (Cronbach alpha $=0.37$ and 0.33 , respectively) was found for diagnostic confidence. We found no significant difference in detection of myelination between clinical and research $\mathrm{T} 2$
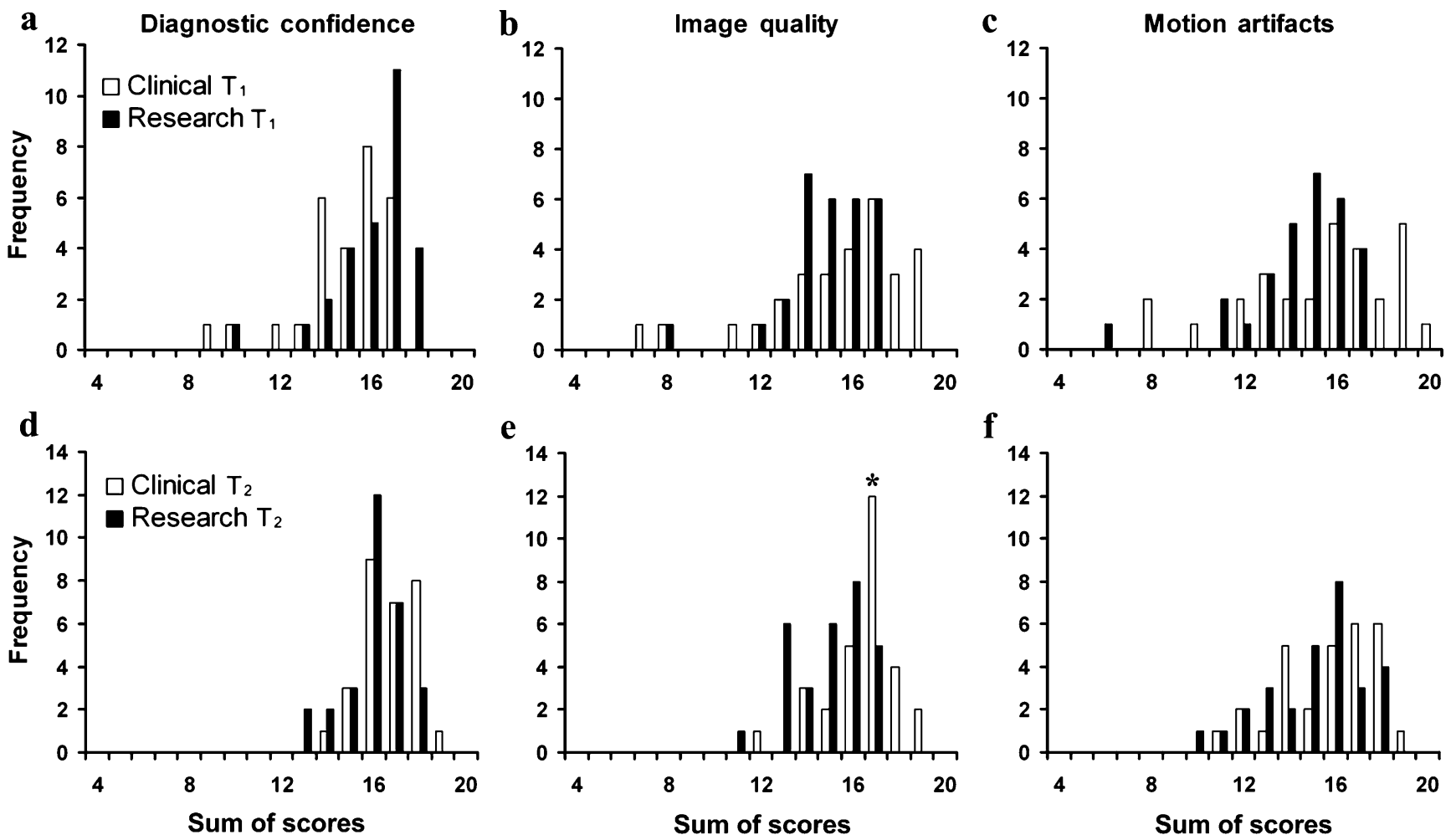

Fig. 3 Histogram of the number of cases for each combined rating result calculated by the sum of scores given by the four raters for each case using a five-level Likert scale for diagnostic confidence, image quality and motion artifacts comparing clinical and research (a-c) T1and (d-f) $\mathrm{T}_{2}-\mathrm{W}$ sequences. $*=$ Significant different combined rating distributions $(P=0.006)$ 
sequences; inter-rater agreement was high for both, in all anatomical regions examined $(\kappa=0.82-1.00)$. Similarly, no significant differences between sequences were found for the detection of sulci. Inter-rater agreement was very high for both clinical and research sequences for all sulci observed $(\kappa \geq 0.86)$ except in the case of the superior temporal sulcus, which produced a kappa of 0.64 for clinical T2, indicating agreement was improved through the use of the research T2 $(\kappa=0.86)$. Separating the data into groups according to radiological findings (normal and non-specific basal ganglia hyperintensity vs. WM injury, GMH I and intraventricular hemorrhage grade II) yielded the same pattern of results for both $\mathrm{T} 1$ and $\mathrm{T} 2$, as no significant differences were found between clinical and research sequences.

\section{Discussion}

Recently there has been an increasing use of $\mathrm{T}_{1}-\mathrm{W}$ and $\mathrm{T}_{2^{-}}$ $\mathrm{W}$ images for brain segmentation in preterm and term neonates [30-37]. Moreover, acquiring volumetric sequences allows for retrospective analyses on the neonates scanned, by having a standardized data set for comparison with future MR studies on the same child. Practical considerations such as minimizing scan time, a lower CNR and the need for confident diagnostic evaluation might hinder the use of high-resolution sequences in clinical settings. Acquisition of conventional and highresolution $\mathrm{T}_{1}-\mathrm{W}$ and $\mathrm{T}_{2}-\mathrm{W}$ sequences to obtain both diagnostic and morphometric information is demanding in time and redundant. Therefore, we compared these two approaches to determine whether high-resolution $\mathrm{T}_{1}-\mathrm{W}$ and $\mathrm{T}_{2}-\mathrm{W}$ images could provide both these values in the very preterm brain.

To properly assess this question, we optimized MR parameters for the high-resolution (research) sequences to balance image resolution, scan time and SNR. As no data were found for preterm neonates scanned at birth at $1.5 \mathrm{~T}$, the experimental parameters optimized for the research $\mathrm{T} 1$ and T2 sequences seen in Table 1 relied on values estimated from the term neonate literature [15, 23, 25]. In parallel, T1 and $\mathrm{T} 2$ values were acquired. Comparing these estimates to the measured values in Table 2, relaxation times of the very preterm brain are, as expected, higher than healthy term neonates scanned at the same field strength. Considering the need to preserve reasonable scan time, these measurements were still consistent with the estimated $\mathrm{T} 1$ values used for calculating the TR/FA combination and the TE used in the research $\mathrm{T} 1$ and $\mathrm{T} 2$ sequences, respectively. Clinical T1 sequences took about 4 min and resulted in a similar SNR and CNR as an optimized turbo SE T1 sequence described in the literature with a similar resolution 
Fig. 4 Histograms of the total score among raters for positive findings (number of raters) confirming myelination detection on clinical and research $\mathrm{T}_{1}-\mathrm{W}$ images in the (a) thalami and (b) cerebellar peduncles. $*=$ Thalamic myelination was significantly better detected on research $\mathrm{T} 1$ sequences $(P=0.02)$

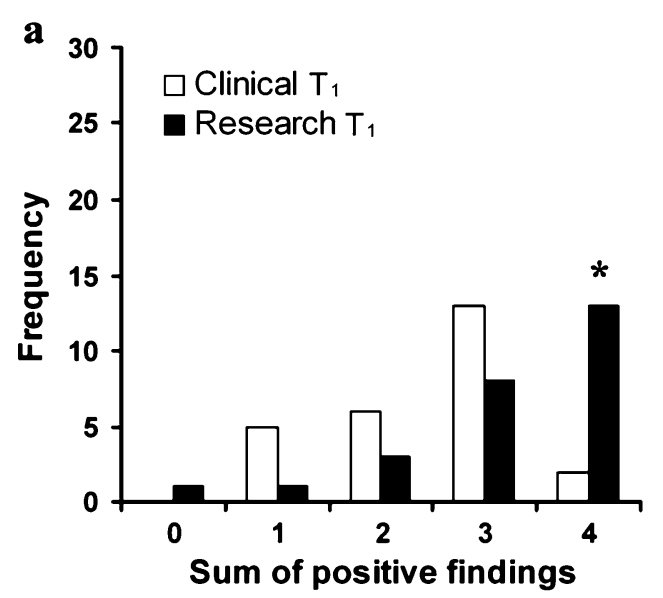

[15]. The $1-\mathrm{mm}^{3}$ resolution research $\mathrm{T} 1$ sequence was obtained with a smaller but adequate SNR and CNR in $5 \min 39 \mathrm{~s}$, similar to an optimized volumetric $\mathrm{T}_{1}-\mathrm{W}$ sequence described in a recent paper having a lower through-plane resolution of $1.5 \mathrm{~mm}$ [18]. Clinical T2 sequences were acquired in both the sagittal and axial planes in a total scan time of $3 \mathrm{~min} 41 \mathrm{~s}$ and high SNR and CNR. Axial clinical T2 scans were obtained using the PROPELLER sequence, which was designed to reduce in-plane rotation and translation head motion and has proved to yield high-quality images with low through-plane resolution in very short scan times [40]. The $1-\mathrm{mm}^{3}$ resolution research $\mathrm{T} 2$ sequence was obtained in $4 \mathrm{~min} 16 \mathrm{~s}$ with a significantly smaller but still adequate SNR and CNR compared to PROPELLER, as seen in Table 1 and Fig. 1. Image quality of clinical protocols might vary among institutions and MRI vendors. Nevertheless, the current research $\mathrm{T} 1$ and $\mathrm{T} 2$ sequences have demonstrated the trade-off between higher resolution and reduced SNR without a significant increase in scan time compared to the chosen clinical protocol, while still maintaining sufficient image quality for diagnostic purposes.

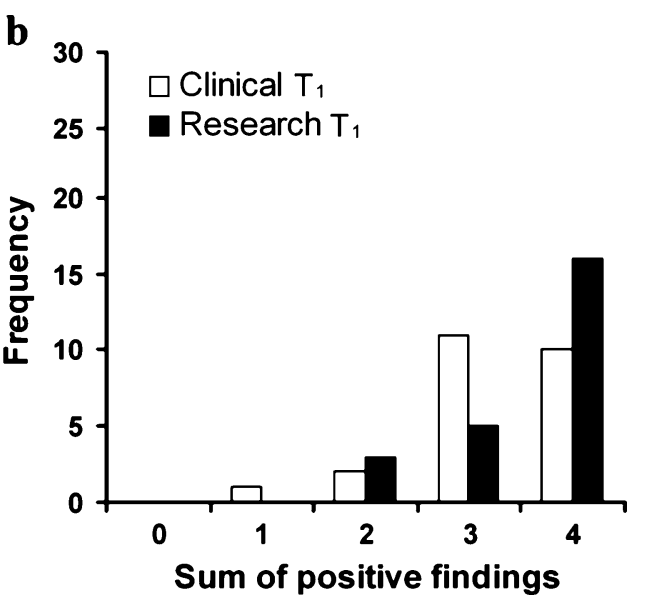

Clinical vs. research high-resolution $\mathrm{T}_{1}-\mathrm{W}$ and $\mathrm{T}_{2}-\mathrm{W}$ sequences

Clinical and research $\mathrm{T} 1$ sequences demonstrated similar performances. There was, however, a trend suggesting higher diagnostic confidence using research $\mathrm{T} 1$ and higher image quality and less motion artifacts for clinical T1. These combined subjective results fit the inherent physical advantages and disadvantages of volumetric gradient echo (GRE) sequences like research T1. Compared to 2-D sequences, volumetric GRE sequences can be acquired with higher resolution for a given SNR and scan time but are more vulnerable to field inhomogeneities, susceptibility and motion artifacts [41]. Because degraded through-plane resolution is less perceptible than degraded in-plane resolution, 2-D imaging protocols are typically designed with higher SNR and CNR at the cost of greater slice thickness. Greater SNR likely explains the preference observed for clinical over research T2 sequences for image quality. Nevertheless, the current analysis indicates no preference between clinical and research sequences for

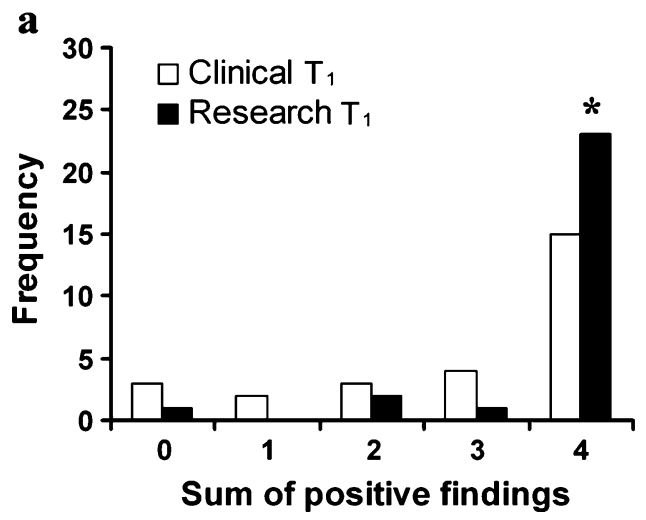

Fig. 5 Histograms of the total score among raters for positive findings (number of raters) indicating the presence of the (a) superior temporal, and $(\mathbf{b})$ calcarine sulcus on clinical and research $\mathrm{T}_{1}-\mathrm{W}$ images. $*=$ b

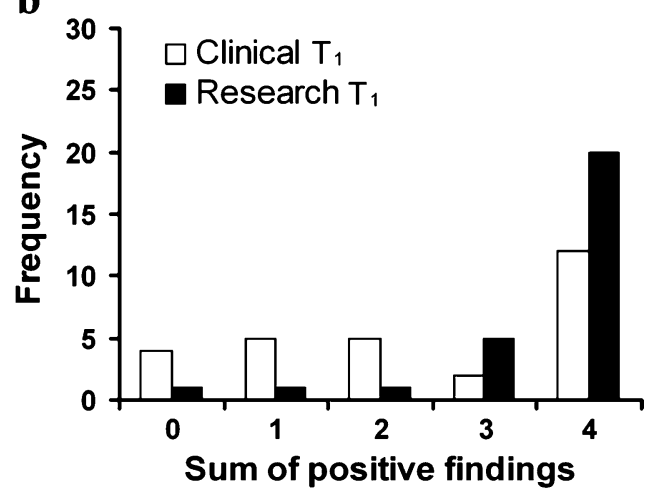

Significant better detection of the superior temporal sulcus was observed on research $\mathrm{T} 1$ sequences $(P=0.03)$ 
extracting diagnostic information. This is likely because clinical sequences have more SNR than necessary to make a diagnosis, while the research sequences effectively convert the excess SNR and in-plane resolution into through-plane resolution, resulting in a detailed image. Although separating the data according to radiological finding did not yield different results, all four raters noted better detection of small pathologies such as punctate white matter lesions on research $\mathrm{T} 1$ images while research $\mathrm{T} 2$ images were found to be useful for detecting small hemorrhages. These findings are in agreement with recent papers proposing the use of volumetric $\mathrm{T}_{1}-\mathrm{W}$ images for clinical evaluation $[12,18]$.

Our results show that high-resolution $\mathrm{T}_{1}-\mathrm{W}$ volumetric imaging shows improved myelination detection compared to conventional $\mathrm{T} 1$ sequences. No difference in myelination detection, however, was found among the T2 sequences. Interestingly, improved consistency, suggesting greater confidence, was demonstrated for $\mathrm{T} 2$ compared to $\mathrm{T} 1$ sequences. The results on the thalamus, particularly, are concordant with previous findings for SE-based sequences of the neonatal brain showing better detection of myelination in GM structures on $\mathrm{T}_{2}-\mathrm{W}$ images, while superior myelination detection in WM structures was observed on $\mathrm{T}_{1}$-W images $[4,6]$. In contrast, in this study, myelination in WM structures was also detected with greater confidence on T2 images. Better detection and improved consistency was found for both superior temporal and calcarine sulci when using research $\mathrm{T} 1$ sequences. This could be explained by increased through-plane resolution yielding greater confidence detection, particularly for the case of small structures, as long as sufficient SNR is preserved $[2,4]$.

\section{Conclusion}

In this study, no significant differences were found between clinical and research $\mathrm{T} 1$ sequences in terms of diagnostic confidence, image quality and motion artifacts. Research $\mathrm{T} 1$ provided better delineation of thalamic myelination and better detection of the superior temporal and calcarine sulci. A clear preference was observed for clinical over research T2 for image quality. Greater confidence was demonstrated for superior temporal sulcus detection with the research sequences. We suggest that high-resolution $\mathrm{T}_{1}-\mathrm{W}$ and $\mathrm{T}_{2}-\mathrm{W}$ volumetric sequences be used for clinical MRI in the very preterm brain to provide both diagnostic and morphometric value and therefore replace currently used conventional imaging sequences.

Acknowledgments This research was supported by the Canadian Institute of Health Research (CIHR MOP-84399). We would like to thank the time and effort spent by our neuroradiologist raters, Drs. M.
M. Shroff, C. Raybaud, J.P. Soares-Fernandes and B. Thomas. We thank MRI technologists Garry Detzler and Ruth Weiss and NICU nurses Angela Thompson and Deborah Singleton, who helped with patient recruitment and were responsible for the well-being of the neonates during scanning.

\section{References}

1. Holland BA, Haas DK, Norman D et al (1986) MRI of normal brain maturation. AJNR 7:201-208

2. Barkovich AJ, Kjos BO, Jackson DE Jr et al (1988) Normal maturation of the neonatal and infant brain: MR imaging at $1.5 \mathrm{~T}$. Radiology 166:173-180

3. van der Knaap MS, van Wezel-Meijler G, Barth PG et al (1996) Normal gyration and sulcation in preterm and term neonates: appearance on MR images. Radiology 200:389-396

4. Barkovich AJ (1998) MR of the normal neonatal brain: assessment of deep structures. AJNR 19:1397-1403

5. Huppi PS, Inder TE (2001) Magnetic resonance techniques in the evaluation of the perinatal brain: recent advances and future directions. Semin Neonatol 6:195-210

6. Counsell SJ, Maalouf EF, Fletcher AM et al (2002) MR imaging assessment of myelination in the very preterm brain. AJNR 23:872-881

7. Counsell SJ, Rutherford MA, Cowan FM et al (2003) Magnetic resonance imaging of preterm brain injury. Arch Dis Child Fetal Neonatal Ed 88:F269-274

8. Barkovich AJ (2005) Pediatric neuroimaging. Lippincott, Philadelphia

9. Dyet LE, Kennea N, Counsell SJ et al (2006) Natural history of brain lesions in extremely preterm infants studied with serial magnetic resonance imaging from birth and neurodevelopmental assessment. Pediatrics 118:536-548

10. Arthur R (2006) Magnetic resonance imaging in preterm infants. Pediatr Radiol 36:593-607

11. Rutherford M, Srinivasan L, Dyet L et al (2006) Magnetic resonance imaging in perinatal brain injury: clinical presentation, lesions and outcome. Pediatr Radiol 36:582-592

12. Barkovich AJ (2006) MR imaging of the neonatal brain. Neuroimaging Clin N Am 16:117-135, viii-ix

13. Nowell MA, Hackney DB, Zimmerman RA et al (1987) Immature brain: spin-echo pulse sequence parameters for high-contrast MR imaging. Radiology 162:272-273

14. Paus T, Collins DL, Evans AC et al (2001) Maturation of white matter in the human brain: a review of magnetic resonance studies. Brain Res Bull 54:255-266

15. Jones RA, Palasis S, Grattan-Smith JD (2004) MRI of the neonatal brain: optimization of spin-echo parameters. AJR 182:367-372

16. Williams LA, DeVito TJ, Winter JD et al (2007) Optimization of 3D MP-RAGE for neonatal brain imaging at 3.0 T. Magn Reson Imaging 25:1162-1170

17. Conklin J, Winter JD, Thompson RT et al (2008) High-contrast 3D neonatal brain imaging with combined T1- and T2-weighted MP-RAGE. Magn Reson Med 59:1190-1196

18. van Wezel-Meijler G, Leijser LM, de Bruine FT et al (2009) Magnetic resonance imaging of the brain in newborn infants: practical aspects. Early Hum Dev 85:85-92

19. Vymazal J, Righini A, Brooks RA et al (1999) T1 and T2 in the brain of healthy subjects, patients with Parkinson disease, and patients with multiple system atrophy: relation to iron content. Radiology 211:489-495

20. Stanisz GJ, Odrobina EE, Pun J et al (2005) T1, T2 relaxation and magnetization transfer in tissue at 3 T. Magn Reson Med 54:507512 
21. Deoni SC, Williams SC, Jezzard P et al (2008) Standardized structural magnetic resonance imaging in multicentre studies using quantitative $\mathrm{T} 1$ and $\mathrm{T} 2$ imaging at $1.5 \mathrm{~T}$. Neuroimage 40:662-671

22. Wright PJ, Mougin OE, Totman JJ et al (2008) Water proton T1 measurements in brain tissue at 7,3, and 1.5 T using IR-EPI, IR-TSE, and MPRAGE: results and optimization. MAGMA 21:121-130

23. Counsell SJ, Herlihy AH, Robertson NJ et al (2000) Elevation of $\mathrm{T} 1$ and $\mathrm{T} 2$ values in the cerebral white matter in periventricular leukomalacia (abstr). The Eighth Annual Meeting of the International Society for Magnetic Resonance in Medicine. Proc Intl Soc Mag Reson Med, Denver, CO, p 1933

24. Counsell SJ, Kennea NL, Herlihy AH et al (2003) T2 relaxation values in the developing preterm brain. AJNR 24:1654-1660

25. Lewis HJ, Allsop JM, Counsell SJ et al (2001) MR quantification of the brain in preterm infants at term equivalent age (abstr). The Ninth Annual Meeting of the International Society for Magnetic Resonance in Medicine. Proc Intl Soc Mag Reson Med, Glasgow, UK p 405

26. Ferrie JC, Barantin L, Saliba E et al (1999) MR assessment of the brain maturation during the perinatal period: quantitative T2 MR study in premature newborns. Magn Reson Imaging 17:12751288

27. Thornton JS, Amess PN, Penrice J et al (1999) Cerebral tissue water spin-spin relaxation times in human neonates at 2.4 tesla: methodology and the effects of maturation. Magn Reson Imaging 17:1289-1295

28. Williams LA, Gelman N, Picot PA et al (2005) Neonatal brain: regional variability of in vivo MR imaging relaxation rates at 3.0 T-initial experience. Radiology 235:595-603

29. Herlihy AH, Counsell SJ, Rutherford MA et al (1999) T1 and T2 measurements of the preterm brain (abstr). The Seventh Annual Meeting of the International Society for Magnetic Resonance in Medicine. Proc Intl Soc Mag Reson Med, Philadelphia, PA, p 531
30. Huppi PS, Warfield S, Kikinis R et al (1998) Quantitative magnetic resonance imaging of brain development in premature and mature newborns. Ann Neurol 43:224-235

31. Prastawa M, Gilmore JH, Lin W et al (2005) Automatic segmentation of MR images of the developing newborn brain. Med Image Anal 9:457-466

32. Xue H, Srinivasan L, Jiang S et al (2007) Automatic segmentation and reconstruction of the cortex from neonatal MRI. Neuroimage 38:461-477

33. Anbeek P, Vincken KL, Groenendaal F et al (2008) Probabilistic brain tissue segmentation in neonatal magnetic resonance imaging. Pediatr Res 63:158-163

34. Dubois J, Benders M, Cachia A et al (2008) Mapping the early cortical folding process in the preterm newborn brain. Cereb Cortex 18:1444-1454

35. Nguyen The Tich S, Anderson PJ, Shimony JS et al (2009) A novel quantitative simple brain metric using MR imaging for preterm infants. AJNR 30:125-131

36. Weisenfeld NI, Warfield SK (2009) Automatic segmentation of newborn brain MRI. NeuroImage 47:564-572

37. Merisaari H, Parkkola R, Alhoniemi E et al (2009) Gaussian mixture model-based segmentation of MR images taken from premature infant brains. J Neurosci Methods 182:110-122

38. Gudbjartsson H, Patz S (1995) The Rician distribution of noisy MRI data. Magn Reson Med 34:910-914

39. Cheng HL, Wright GA (2006) Rapid high-resolution T(1) mapping by variable flip angles: accurate and precise measurements in the presence of radiofrequency field inhomogeneity. Magn Reson Med 55:566-574

40. Pipe JG (1999) Motion correction with PROPELLER MRI: application to head motion and free-breathing cardiac imaging. Magn Reson Med 42:963-969

41. Haacke EM, Brown RW, Thompson MR et al (1999) Magnetic resonance imaging: physical principles and sequence design. Wiley, Toronto 